\title{
Optimal Controller for Near-Space Interceptor with Actuator Saturation
}

\author{
FAN Guo-long** \\ Northwestern Polytechnic University, Xi'an, China \\ LIANG Xiao-geng**
}

China Airborne Missile Academy, Luoyang, China

\section{HOU Zhen-qian*** and YANG Jun****}

Northwestern Polytechnic University, Xi'an, China

\begin{abstract}
The saturation of the actuator impairs the response performance of the near space interceptor control system. A control system based on the properties of linear tracking system is designed for this problem. The properties are that the maximum value of the pseudo-Lyapunov function of the linear tracking control system do not present at the initial state but at the steady state, based on which the bounded stability problem is converted into linear tracking problem. The pseudo-Lyapunov function of the linear tracking system contain the input variables; the amplitude and frequency of the input variables affect the stability of the nonlinear control system. Designate expected closed-loop poles area for different input commands and obtain a controller which is function of input variables. The coupling between variables and linear matrices make the control system design problem non-convex. The non-convex problem is converted into a convex LMI according to the Shur complement lemma and iterative algorithm. Finally the simulation shows that the designed optimal control system is quick and accurate; the rationality of the presented design techniques is validated.
\end{abstract}

Key words: Actuator Saturation, the bounded stability, Optimal Controller

\section{Nomenclature}

$\begin{array}{ll}e & \text { Integration of overload error } \\ \alpha & \text { Angle of attack of interceptor } \\ \omega_{z} & \text { Pitch angle rate of interceptor } \\ a_{i j} & \text { Aerodynamic force coefficients and aerodynamic } \\ & \text { moment coefficients } \\ v & \text { Speed of interceptor } \\ \mathrm{g} & \text { Acceleration of gravity } \\ n_{\mathrm{zc}} & \text { Command of overload } \\ A & \text { State matrix } \\ B & \text { Control matrix } \\ x & \text { System state vector } \\ u & \text { Control input vector }\end{array}$

This is an Open Access article distributed under the terms of the Creative Commons Attribution Non-Commercial License (http://creativecommons.org/licenses/by$\mathrm{nc} / 3.0 /$ which permits unrestricted non-commercial use, distribution, and reproduction in any medium, provided the original work is properly cited.

\begin{tabular}{|c|c|}
\hline$K$ & State feedback controller \\
\hline Sat(.) & Saturation limitation \\
\hline$\rho$ & $\begin{array}{l}\text { Saturation value of the Rudder deflection angle } \\
\text { or angle rate }\end{array}$ \\
\hline$P, W, G, H$ & Positive definite matrices \\
\hline$V(x)$ & the pseudo-Lyapunov function \\
\hline$A_{c}$ & Closed loop system matrix \\
\hline$\psi(\cdot)$ & Decentralized dead-zone nonlinearity \\
\hline$\lambda()$. & Eigenvalue of matrix \\
\hline
\end{tabular}

\section{Introduction}

The actuator nonlinear characteristics of tactical missile in aerosphere can be ignored when designing the control 
system because of the natural frequency of missile is far higher than $1 \mathrm{~Hz}$, the actuator can be regarded as first-order inertial module[1-2]. However, the natural frequency of the near space intercept missile is lower than $1 \mathrm{~Hz}$. A higher gain controller is needed to enhance the response speed of the interceptor control system. With the increase of the flight altitude, the maneuverability of interceptor is decreased because of the saturation nonlinear characteristics of the actuator, so it is difficult to design the linear control system with actuator saturation constraints for the near space intercept missile that can track the command more quickly and accurately.

The tracking control system design is one of the most popular control problems in engineering[7-9], including accurate tracking based on model reference adaptive control[3-4] and the standard optimal tracking control (the reference input signals are regarded as the disturbance signals[5-6]), but they ignored the nonlinear of the actuator. The neglected actuator saturation is the source of limit cycle, parasitic equilibrium points and even instability of the closed loop system[5-6]. David h. Klyde[10] pointed out that when the actuator has saturation phenomena, the stability of the control system dependent on the input variables. It is considered that the controller is a function of the input variables to maintain the stability of the control system and improve the time response speed of the nonlinear system.

Section 2 introduces the integration of overload error , given the classic three-loop autopilot structure and establishment of the linear tracking system mathematical model. Section 3 presents the properties of the linear tracking system in which the maximum value of the pseudo-Lyapunov function for linear tracking system appeared at the steady states instead of the initial states based on Bounded stability theory. Section 4 extends this character to the case when the linear control system with control input saturation constraints, presents the controller as a function of input variables. According to the Shur complement lemma and iterative algorithm, the non-convex problem is converted into a convex LMI. Simulation results are presented in Section 5 to demonstrate the methods, and Section 6 presents concluding remarks.

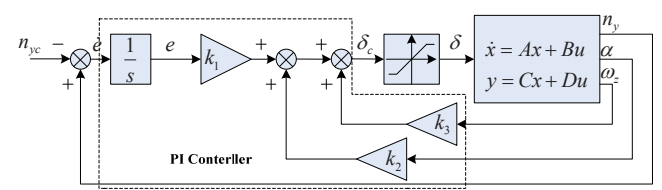

Fig. 1. The autopilot PI control structure of the classic three loops

\section{The Interceptor Model and Autopilot Structure}

Ignoring the dynamic characteristics of the actuator, the longitudinal state space model of the interceptor is given:

$$
\left[\begin{array}{c}
\dot{\alpha} \\
\dot{\omega}_{z}
\end{array}\right]=\left[\begin{array}{cc}
-a_{34} & 1 \\
-a_{24} & -a_{22}
\end{array}\right]\left[\begin{array}{c}
\alpha \\
\omega_{z}
\end{array}\right]+\left[\begin{array}{l}
-a_{35} \\
-a_{25}
\end{array}\right] \delta_{c}
$$

Where $\alpha$ - Angle of attack of interceptor; $\omega_{z}$ - Pitch angle rate of interceptor; $a_{i j}$-Aerodynamic force coefficients and aerodynamic moment coefficients.

The classic three loops autopilot structure and the mathematical model are given. The autopilot structure is shown in Fig.1, in which the pitch angle rate feedback loop can upgrade the low damping of the missile; the pseudo Angle of attack feedback loop can improve the static instability of the missile and increase time response. The overload error integral variables are introduced to realize accurate tracking of the instructions $[8,12]$ :

$$
e=\int_{0}^{t}\left(n_{z}-n_{z c}\right) d t \Rightarrow \dot{e}=\frac{a_{34}}{g} v+\frac{a_{35}}{g} v-n_{z c}
$$

Remark1: in Fig. 1 the command of overload regarded as the input signals, the controller are proportion and integration controller; in Fig. 2 the command of overload and the integration controller regarded as the systems model, the controller is pure proportion controller. Integration Control of overload error converted into proportion control of overload error integration. The yield static output feedback control; this can resort to solving LMIs.

From the above equation (1) (2), the augmented state equation is established:

$$
\left[\begin{array}{c}
\dot{e} \\
\dot{\alpha} \\
\dot{\omega}_{z}
\end{array}\right]=\left[\begin{array}{ccc}
0 & a_{34} v / g & 0 \\
0 & -a_{34} & 1 \\
0 & -a_{24} & -a_{22}
\end{array}\right]\left[\begin{array}{c}
e \\
\alpha \\
\omega_{z}
\end{array}\right]+\left[\begin{array}{c}
a_{35} v / g \\
-a_{35} \\
-a_{25}
\end{array}\right] \delta_{c}+\left[\begin{array}{c}
-1 \\
0 \\
0
\end{array}\right] n_{z c}=A x+B u+E n_{z c}
$$

Where: $v$ - Speed of the interceptor; g- Acceleration of gravity; $n_{z c}$ - Command of the overload.

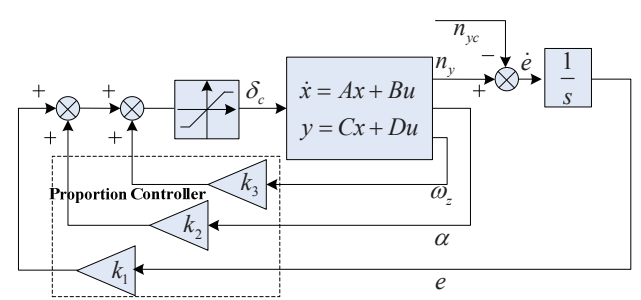

Fig. 2. The autopilot proportion control structure of the three loops 


\section{The Function Properties of Linear Tracking System}

\subsection{Preliminaries}

Introducing state feedback control for plant of equation (3):

$$
u(t)=\operatorname{sat}(K x(t))
$$

Where each component of the control vector, given by $u_{i}(t)=\operatorname{sat}\left(K_{i} x(t)\right), \forall i=1, \cdots, m$, is defined as [5]:

$$
u_{i}(t)=\left\{\begin{aligned}
\rho_{i} & \text { if } K_{i} x(t) \geq \rho_{i} \\
K_{i} x(t) & \text { if }-\rho_{i}<K_{i} x(t)<\rho_{i} \\
-\rho_{i} & \text { if } K_{i} x(t) \leq-\rho_{i}
\end{aligned}\right.
$$

The closed-loop system can be represented as:

$$
\dot{x}(t)=A_{c} x(t)-B \psi(K x(t))+E n_{z c}
$$

Where $A_{c}=A+B K$, and

$$
\psi(K x(t))=K x(t)-\operatorname{sat}(K x(t))
$$

is a decentralized dead-zone nonlinearity.

Now, consider a matrix $G \in R^{m \times n}$ and define the set:

$$
\Sigma=\left\{x \in R^{m \times n}:\left|\left[K_{i}-G_{i}\right] x\right| \leq \rho_{i} \quad i=1, \cdots, m\right\}
$$

Theorem 1 [11]: for all $x \in \Sigma$, with $\psi(K x(t))$ given by (7) that

$$
\psi^{T}(K x(t)) T(\psi(K x(t))-G x(t)) \leq 0
$$

for any positive definite diagonal matrix $T \in R^{m \times m}$.

Lemma 1[13]: The following statements are equivalent:

1. $n$ order matrix $F$ is stability.

2. If there exist Hermit positive definite matrices $G$ and $H$, such that $F=G H$.

Theorem 2 [11]: If the $n$-order real matrix $A$ and $B$ are symmetric positive definite matrix, then all of the eigenvalues

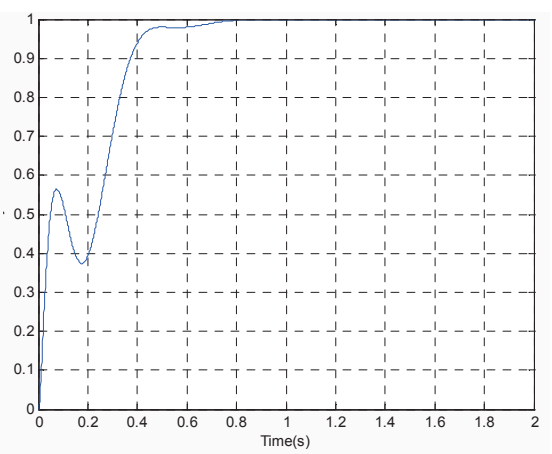

Fig. 3. The normal overload response of the $A B$ are greater than zero.

The next theorem is given based on theorm1 2 and the bounded stability theory.

Theorem 3: The time-derivative of the pseudo-Lyapunov function for the linear tracking system is nonnegative, when time tends to infinity, the time-derivative of the function is null. That is to say: the maximum of the function of the tracking system is not in the initial state but in the steady state.

Proof: Choice the function as $V(x)=x^{T} P x\left(P^{T}=P, P>0\right)$, the time-derivative of the function along the system trajectories (6) is given by

$$
\dot{V}(x)=x^{T}\left(A_{c}^{T} P+P A_{c}\right) x+n_{z c} E^{T} P x+x^{T} P E n_{z c}
$$

The solutions of the first-order linear time-invariant differential equations $\dot{x}(t)=A_{c} x(t)+E n_{z c}$ :

$$
x(t)=e^{A_{c} t} x(0)+\int_{0}^{t} e^{A_{c}(t-\tau)} E n_{z c} d \tau
$$

The solution for the zero initial conditions of the system is:

$$
x(t)=n_{z c}\left[e^{2 A_{c} t}-e^{A_{c} t}\right] A_{c}^{-1} E
$$

Taking into account (10), that:

$$
\dot{V}(x)=n_{z c}^{2} E^{T}\left\{\begin{array}{l}
\left(\left[e^{2 A_{c} t}-e^{A_{c} t}\right] A_{c}^{-1}\right)^{T}\left(A_{c}^{T} P+P A_{c}\right)\left[e^{2 A_{c} t}-e^{A_{c} t}\right] A_{c}^{-1} \\
+P\left(\left[e^{2 A_{c} t}-e^{A_{c} t}\right] A_{c}^{-1}\right)+\left(\left[e^{2 A_{c} t}-e^{A_{c} t}\right] A_{c}^{-1}\right)^{T} P
\end{array}\right\} E .
$$

The positive and negative of $\dot{V}(x)$ is equivalent to the positive and negative of matrix $\Delta$ :

$$
\Delta=A_{c}^{-T}\left\{\left[e^{2 A t}-e^{A t}+I\right]^{T} A_{c}^{T} P\left[e^{2 A t}-e^{A t}\right]+\left[e^{2 A t}-e^{A_{t} t}\right]^{T} P A_{c}\left[e^{2 A t}-e^{A t}+I\right]\right\} A_{c}^{-1}
$$

As $\lambda\left(-A_{c}\right)>0$, by the lemma 1 and theorem 2 , that $\lambda(\Delta) \geq 0 \Rightarrow \dot{V}(x) \geq 0$.

Namely, the time-derivative of the function of the linear tracking system is greater than or equal to zero, $\dot{V}(x)=0$, when $\mathrm{t} \rightarrow \infty$.

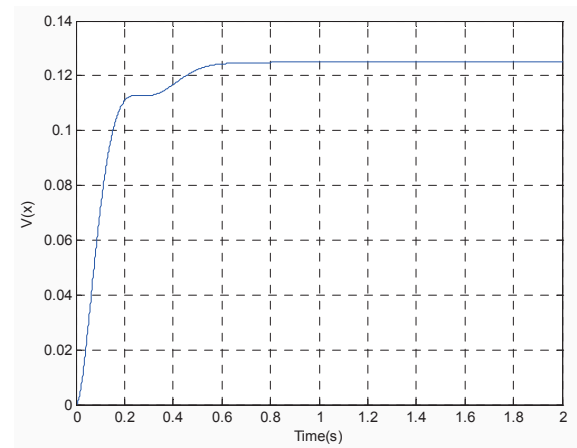

Fig. 4. The value of Lyapunov function 


\subsection{Numerical experiments}

We give an example to validate that the time-derivative of the function of the linear tracking system is nonnegative to illuminate the above Theorem 3 visually.

If there exist positive definite symmetric matrix $W \in R^{n \times n}$, matrix $Z \in R^{m \times n}$ and constants $\mu, \tau, \sigma \in R$, satisfy the following LMIs :

$$
\left\{\begin{array}{c}
A W+W A^{T}+B Z+B^{T} Z^{T}+2 \mu W \leq 0 \\
{\left[\begin{array}{cc}
2 \mu W & -E \\
-E^{T} & \sigma r^{-2}
\end{array}\right] \geq 0} \\
A W+W A^{T}+B Z+B^{T} Z^{T}+2 \mu W+\tau W \geq 0
\end{array}\right.
$$

then the controller is as follows:

$$
K=Z W^{-1}
$$

The eq(14) are the simplified presentation of Theorem 4 under the conditions of a linear system. The proof progress see Theorem 4 , the maximum value of the system function appears in steady state, and the value is $L=\sigma / \mu$, here $\mu$ is the desired closed-loop system power exponential convergence rate.

Example: given state matrix and control matrix of an aircraft at a typical flight point as follows:

$$
A=\left[\begin{array}{ccc}
0 & 5.78 & 0 \\
0 & -0.06 & 1 \\
0 & -7.11 & 0
\end{array}\right], B=\left[\begin{array}{c}
0.73 \\
-0.008 \\
13.33
\end{array}\right]
$$

Let the negative real part of desired closed-loop pole $\mu$ $=-8$, and let $\tau=0.0001, \sigma=1$, according to equation (14), we search for $\lambda(W)$, then the following controller is obtained: $\mathrm{K}=\left[\begin{array}{lll}-24.9446 & -28.2807 & -0.4504\end{array}\right]$.

When the input command is $n_{z c}=1$, Fig. 3 shows normal overload response curve, Fig. 4 shows the function value of the tracking system varies with time.

Through the simulation it is concluded that: (1) in the dynamic adjustment process, the function value for the linear tracking system increases with the simulation time, when the system states are steady, the function value reach its maximum value, just as theorem 3 said; (2) the designed control system track the instruction quickly and accurately; (3) the maximum value of the function is close to $\sigma / \mu=0.125$.

\section{Control System Design and Linearization}

\subsection{Control system design}

Theorem 4: For any constant matrices controllable pair $(A, B)$ with appropriate size, if there is a positive definite symmetric matrix $W \in R^{n \times n}$, matrices $Z \in R^{m \times n}, \quad Y \in R^{m \times n}$ diagonal matrix $S \in R^{m \times m} \mu, \tau \in R, v(t) \in\left[\begin{array}{ll}1 & \infty\end{array}\right)$ and a constant matrix $\kappa, \sigma, \rho_{i} \in R$, that:

$$
\begin{aligned}
& {\left[\begin{array}{cc}
W & * \\
Z_{i}-Y_{i} & \rho_{i}^{2} \mu / \sigma
\end{array}\right] \geq 0, i=1, \cdots, m} \\
& A W+W A^{T}+B Z+Z^{T} B^{T}+2 \mu W \leq 0 \\
& {\left[\begin{array}{cc}
A W+W A^{T}+B Z+Z^{T} B^{T}+\tau W+2 \mu W & * \\
-S B^{T}-Y & 2 S
\end{array}\right] \geq 0} \\
& {\left[\begin{array}{cc}
2 \mu W & * \\
-E^{T} & \sigma n_{z c}^{2}
\end{array}\right] \geq 0}
\end{aligned}
$$

$$
\left[\begin{array}{cc}
v(t) \kappa^{2} \rho_{i}^{2} W & * \\
Z_{i} & \mu / \sigma
\end{array}\right] \geq 0, i=1, \cdots, m
$$

Where $n_{z c}$ is overload command, $\rho_{i}$ is the value of the rudder saturation and $E=\left[\begin{array}{lll}-1 & 0 & 0\end{array}\right]^{T}$.

The controller gain is:

$$
K=Z W^{-1}
$$

Closed loop system is asymptotically stable in the area of the ellipsoidal region $\Xi \triangleq\left\{x \mid x^{T} P x \leq \sigma / \mu\right\}$, and the control system input command $|K x| \leq \kappa \rho$.

Proof: Pre and post-multiplying (14) by diagonal matrix $\operatorname{diag}\left\{W^{-1}, I\right\}$, and taking into account the variable transformations $Z=K W, Y=G W, W^{-1}=P$ :

$$
\left[\begin{array}{cc}
P & * \\
K_{i}-G_{i} & \rho_{i}^{2} \mu / \sigma
\end{array}\right] \geq 0, i=1, \cdots, m
$$

Introduction of Shur complement lemma and the (8) ensures that $\Xi \subseteq \Sigma$.

The response of closed-loop system converges to input command in form of power exponent through (14).

Suppose there exist $\tau$ satisfying $-x^{T} \tau P x \leq \dot{V}(x)$, and taking into account (6):

$$
x^{T}\left(A_{c}^{T} P+P A_{c}\right) x-(B \psi)^{T} P x-x^{T} P B \psi+n_{z c} E^{T} P x+x^{T} P E n_{z c}+x^{T} \tau P x \geq 0
$$

By the function properties of the linear tracking system and (15):

$$
2 x^{T} \mu P x \leq n_{z c} E^{T} P x+x^{T} P E n_{z c}
$$

Then put (22) into (21) and applying the Schur complement lemma:

$$
\left[\begin{array}{cc}
A_{c}^{T} P+P A_{c}+\tau P+2 \mu P & -P B \\
-B^{T} P & 0
\end{array}\right] \geq 0
$$

Then put (8) into (23): 


$$
\left[\begin{array}{cc}
A_{c}^{T} P+P A_{c}+\tau P+2 \mu P & -P B-G^{T} T \\
-B^{T} P-T G & 2 T
\end{array}\right] \geq 0
$$

Pre and post-multiplying (23) by diagonal matrix $\operatorname{diag}\{W$, $S\}$, and taking into account the variable transformations $P=W^{-1}, S=T^{-1}$, it follows that (16).

Suppose there exist $\sigma$ satisfying the following condition:

$$
n_{z c} E^{T} P x+x^{T} P E n_{z c} \leq 2 x^{T} \mu P x+\sigma
$$

The vector form of (25) can be rewritten as:

$\left[\begin{array}{cc}x^{T} & n_{z c}^{T}\end{array}\right]\left\lfloor\begin{array}{cc}2 \mu P & * \\ -E^{T} P & \sigma n_{z c}^{-2}\end{array}\right\rfloor\left\lfloor\begin{array}{c}x \\ n_{z c}\end{array}\right\rfloor \geq 0$

The inequality is equivalent to:

$$
\left[\begin{array}{cc}
2 \mu P & * \\
-E^{T} P & \sigma n_{z c}^{-2}
\end{array}\right] \geq 0
$$

Through state transitions (27) is equivalent to (17).

According to the norm of the definition that $\left|K_{i} x\right| \leq \kappa \rho$ can be rewritten as:

$$
x^{T} K_{i}^{T} K_{i} x \leq \kappa^{2} \rho^{2}
$$

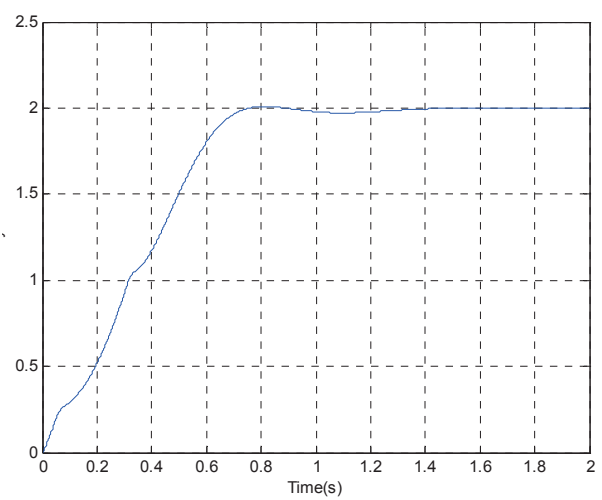

Fig. 5. The normal acceleration response curve (Typical point 1)

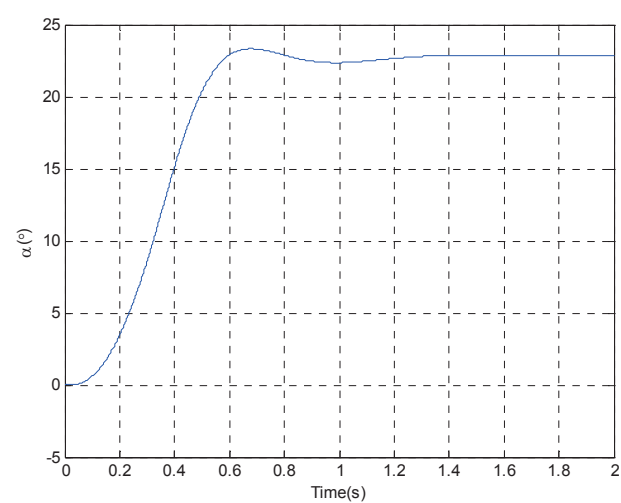

Fig. 6. The attack angle response curve (Typical flight point 1)
According to the function properties of the linear tracking system, suppose there exist $v(t) \in\left[\begin{array}{ll}1 & \infty\end{array}\right]$ satisfying the following condition:

$$
v(t) x^{T}(t) P x(t)=\sigma / \mu
$$

Then:

$$
v(t) \kappa^{2} \rho^{2} P-\sigma / \mu Z_{i}^{T} P P Z_{i} \geq 0
$$

Applying the Schur complement lemma and state transform that (30) is equivalent to (18).

\subsection{Linearization of the control system}

We discuss now how to compute the matrices gain $\mathrm{K}$ from the conditions stated in theorem 4 . The variables to be found by applying theorem 4 are $W, Z, Y, S, \mu, \tau, \nu, \kappa, \sigma, \rho_{i}$. Since the inequalities terms involving the product between these variables, inequalities (15) (18) are nonlinear matrix inequalities. To convert the non convex optimization problem into a convex optimization problem, an iterative algorithm is given as follows.

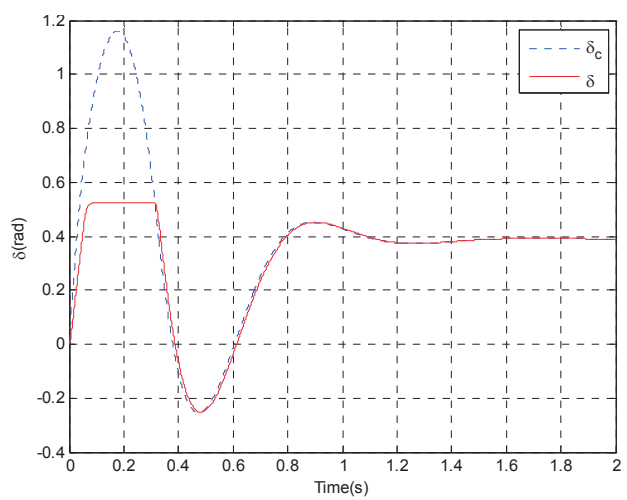

Fig. 7. The rudder angle response curve (Typical point 1)

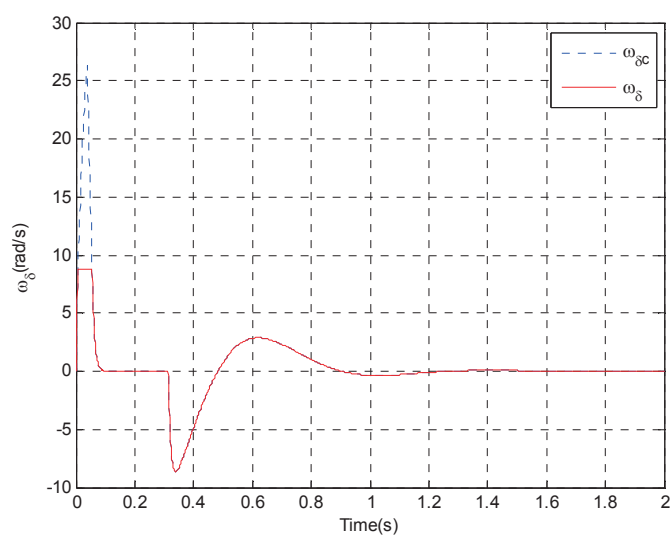

Fig. 8. The rudder angle rate response curve (Typical point 1) 
First: according to the ideal frequency response of the closed-loop system, fix the value $v(t)=v_{0}, \kappa=\kappa_{0}$.

Second: given the initial value $\mu=\mu_{0}, \tau=\tau_{0}$, in this case, the matrix inequalities of theorem 4 become LMIs. The eigenvalues of the variable $W$ can be minimized by solving the convex optimization problem, to enhance the time response speed of the control system:

$$
\left\{\begin{array}{c}
\min \lambda(W) \\
s t: \operatorname{LIMs}(14) \sim(18)
\end{array}\right.
$$

Third: Fixed $W=W_{0}$, the matrix inequalities of the theorem 4 (14) (18) are converted into LMIs, The variables value of the $\mu, \tau$ can be minimized by solving the following convex optimization problem with LMI constraints:

$$
\left\{\begin{array}{c}
\min (-\alpha \mu+(1-\alpha) \tau) \\
s t: \operatorname{LIMs}(14) \sim(18)
\end{array}\right.
$$

In each step, some variables are fixed and a convex optimization problem with LMI constraints is solved:

Step 0: Initialize $v(t)=v_{0}, \kappa=\kappa_{0}, \mu=\mu_{0}, \tau=\tau_{0}$, according to the ideal frequency response of the closed-loop system.

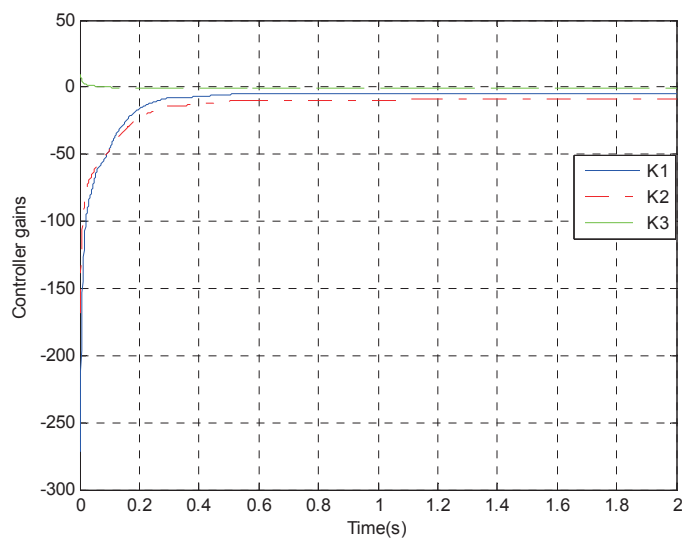

Fig. 9. The controller gains curve according to time (Typical point 1)

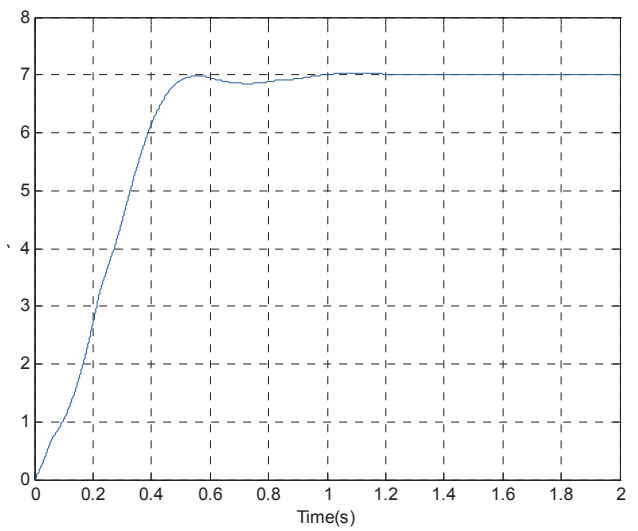

Fig. 10. The normal acceleration response curve (Typical point 2)
Step 1: solve (31) for $W$.

Step 2: fixed value $W$, solve (32) for $\mu$ 、 $\tau$.

Step 3: take the positive value of $\Delta \mu$ such that $\Delta \mu<<\mu$, let $\mu=\mu+\Delta \mu$ back to step 1 until no significant change in the optimal value of $\mu$ occurs.

The iteration between these three steps stops when a desired precision for $\mu$ is achieved.

Remark2: When $v_{0}=1$ the actuator of the control system does not appear the saturation phenomenon, when $v_{0}>>1$ the actuator of the control system appear the saturation phenomenon.

\section{Simulation and Results}

Typical flight point 1 : The flight height of the interceptor is $H=33 \mathrm{~km}$, the speed is $3.5 \mathrm{Ma}$, Angle of attack is $20 \mathrm{deg}$, the rudder deflection angle saturation is $\pm 30^{\circ}$ and the rudder deflection angle rate saturation is $\pm 500^{\circ} / \mathrm{s}$. When normal acceleration command is $2 \mathrm{~g}$, Fig. 5 show the overload response curve, Fig. 6 show the Angle of attack response

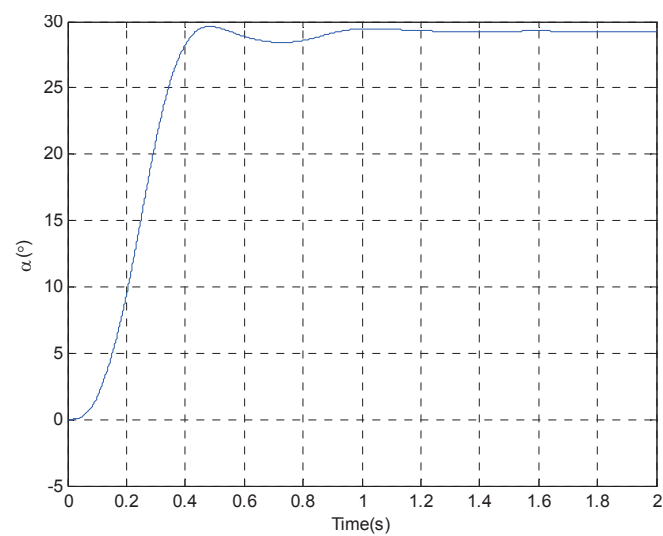

Fig. 11. The attack angle response curve (Typical point 2)

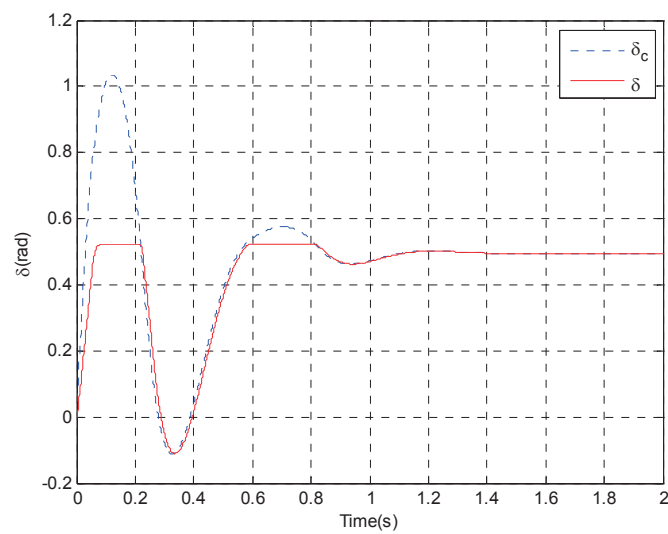

Fig. 12. The rudder angle response curve (Typical point 2) 
curve, Fig. 7 show the rudder angle response curve, Fig. 8 show the rudder angle rate response curve, and Fig. 9 shows the controller gains curve according to time.

Typical flight point 2: The flight height of the interceptor is $H=30 \mathrm{~km}$, the speed is $4 \mathrm{Ma}$, angle of attack is $20 \mathrm{deg}$, and the rudder deflection Angle saturation is $\pm 30^{\circ}$ and the rudder deflection angle rate saturation is $\pm 500 \%$ s. When normal acceleration command is $7 \mathrm{~g}$, Fig.10 show the overload response curve, Fig.11 show the Angle of attack response curve, Fig. 12 show the rudder angle response curve, Fig.13 show the rudder angle rate response curve, and Fig.14 shows the controller gains curve according to time.

The simulation results of the linear control system with actuator saturation constraints show that with the increase of altitude and reduce of flight Mach, the time response speed of the control system and the ability of tracking the normal acceleration command significantly deteriorate. The Controller is a function of the input command, so fast tracking of input command can be realized.

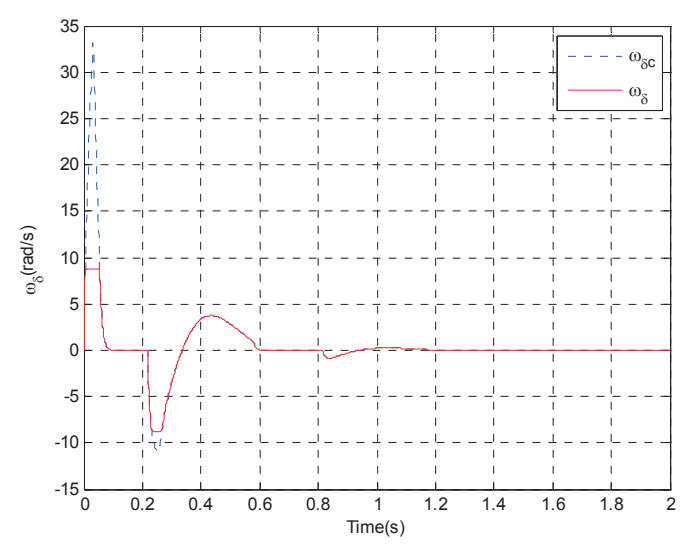

Fig. 13. The rudder angle rate response curve (Typical point 2)

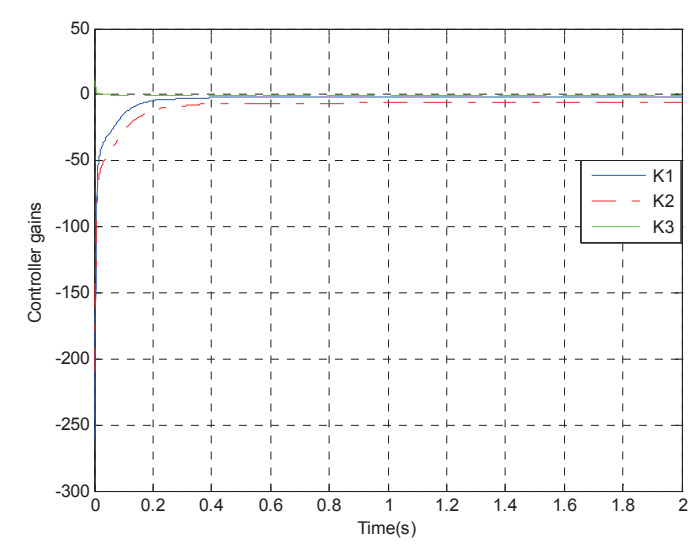

Fig. 14. The controller gains curve according to time (Typical point 2)

\section{Conclusions}

The design control system of the near space intercept missile makes it track the command more quickly and accurately when the control input has saturation constraints. We described the classic three-loop autopilot structure and the linear tracking system mathematical model, in which Integration Control of the overload error is converted into proportion control of the overload error integration, which yields static output feedback control, this can resort to solving LMIs. We present the maximum value of the pseudoLyapunov function for the linear tracking system (theorem 3). This appeared at the steady states instead of the initial states based on theorem 1 2 and the Bounded stability theory. The time-derivative of the function (e.g. (10)) contains the input command, so the amplitude and frequency of the command affect the stability of nonlinear control system, designate expected closed-loop poles area for different input commands and obtain a controller which is function of input variables. The coupling between the variables and linear matrices $((15) \sim(18))$ make the control system design problem non-convex. The non-convex problem is converted into a convex LMI according to the Shur complement lemma and iterative algorithm. Finally two examples of the typical flight points verify the effectiveness of the presented design technique. The proposed design method can be applied to the horizontal lateral-directional system model and arbitrary controllable system.

\section{References}

[1] XING Li-dan, CHEN Wan-chun, "Autopilot Design for Missile Controlled by Lateral Thrust/Aero Force using Combined Optimal/Classical Approach", Journal of Beijing University of Aeronautics and Astronautics, Vol.35, No.08, pp.921-924.

[2] CHANG Chao, LIN De-fu, "Pole Assignment Method with State Constraints for Missile Three-loop Autopilot Design", Chinese Control and Decision Conference, Guilin, 2009, pp. 3298-3301.

[3] ZHANG Wei-guo, LIU Xiao-xiong, "Design of a Robust Adaptive Reconfigurable Flight Control System Using Multiple Controller", Journal of Northwestern Polytechnical University, Vol.25, No.1, pp.103-107.

[4] Nishant Unikrishnan, "Missile Longitudinal Autopilot Design Using a New Model-Following Robust NeuroAdaptive Controller", AIAA Guidance Navigation and Control Conference and Exhibit, Rhode island, 2004, pp.1-12.

[5] FEN Wu, K.M. Grigoriadis, "LPV Based Control of 
Systems with Amplitude and Rate Actuator Saturation Constraints", Proceedings of the American control Conference, Houston, 1999, pp.3191-3195.

[6] Joā̄ M. Gomes Da Silva Jr, Sophie Tarbouriech, "Local Stabilization of Linear Systems under Amplitude and Rate Saturating Actuators", Proceedings of the $39^{\text {th }}$ IEEE Conference on Decision and Control, Sydney, 2000, pp.3260-3265.

[7] CHEN Shan-ben, ZHANG Quan, "A Time-Domain Approach to $\mathrm{H}_{\infty}$ Optimal Tracking Problem of the Systems with L2 Bounded Uncertainty Disturbances", Journal of Astronautics, Vol.16, No.3, pp.25-31.

[8] ZHANG Qing-jiang, YE Si-jun, LI Yan, WANG Xinmin, "An Enhanced LMI Approach for Mixed H2/Hळ Flight Tracking Control”, Chinese Journal of Aeronautics, Vol.24, No.3, pp.324-328.

[9] ZHANG Huaiquan, CHEN Shuanyong, "Electric Power Steering Simulation Analyze Based on Fuzzy PID Current Tracking Control", Journal of Computational Information Systems, Vol.7, No.1, pp.119-126.

[10] David H. Klyde, Duane T. McRuer, Thomas T.
Myers, "PIO Analysis with Actuator Rate Limiting", AIAA Atmospheric Flight Mechanics Conference, San Diego, 1996, pp.569-580.

[11] Akin Delibasi, Ibrahim B. Kucukdemiral, and Galip Cansever, "L2 control of LPV systems with saturating actuators: Polya approach", Optimal Control Applications and Methods, Vol.34, pp.17-34.

[12] Jinhua Fan, Zhiqiang Zheng, Youmin Zhang, "Faulttolerant Control for Output Tracking Systems Subject to Actuator Saturation and Constant Disturbances: an LMI Approach", AIAA Guidance, Navigation, and Control Conference, Oregon, 2011-6411.

[13] YONG Xue-rong, "The H-, Positive Definite-, Positive Stable-, and P-Matrices", Journal of Xi'an Jiaotong University, Vol.31, No.4, pp.103-107.

[14] TANG Jian-guo, "Upper and Lower Bound of Eigenvalues for Multiplication of Two Real Symmetric Matrices", Journal of Yanbian University, Vol.35, No.4, pp.302-304. 